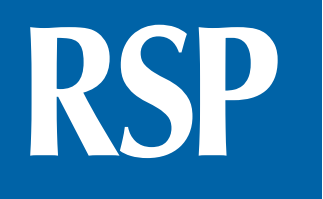

http://www.rsp.fsp.usp.br/
Revista de Saúde Pública

\title{
Trayectorias de estrés familiar y laboral y su asociación con accidentes cerebrovasculares
}

\author{
María Pilar Jerez' (iD, Ignacio Madero-Cabib ${ }^{11, \text { III }}$ \\ Magíster en Salud Pública. Pontificia Universidad Católica de Chile. Santiago, Chile \\ " Pontificia Universidad Católica de Chile. Instituto de Sociología \& Departamento de Salud Pública. Santiago, Chile \\ III Millennium Nucleus for the Study of the Life Course and Vulnerability (MLIV). Santiago, Chile
}

\section{RESUMEN}

OBJETIVOS: Reconstruir tipos de trayectorias de estrés simultáneo en el dominio familiar y laboral en diferentes etapas de la vida y estimar su asociación con accidentes cerebrovasculares (ACV) en la vejez.

MÉTODOS: Se utilizó una encuesta retrospectiva, cara a cara, y representativa de personas de 65 a 75 años en la ciudad de Santiago de Chile $(\mathrm{n}=802)$. Se empleó un análisis de secuencias multicanal para reconstruir tipos de trayectorias de estrés familiar y laboral en diversas etapas de la vida y luego se utilizaron modelos de regresión logística para estimar la asociación de estos tipos de trayectoria con ACV en la vejez, controlado por factores tradicionales de riesgo cardiovascular.

RESULTADOS: Se identificaron cuatro tipos representativos de trayectorias de estrés familiar y laboral: (1) Ausencia de estrés familiar y laboral, (2) Ausencia de estrés familiar, estrés laboral persistente, (3) Ausencia de estrés familiar, fuera de mercado laboral, y (4) Estrés familiar persistente, ausencia de estrés laboral. El 61,7\% de la muestra siguió trayectorias marcadas por la presencia permanente de estrés familiar y/o laboral. Asimismo, el 18,3\% tuvo una trayectoria caracterizada por la ausencia prolongada del mercado del trabajo. Las personas con trayectorias de estrés familiar o laboral persistente, así como aquellas con períodos extensos de inactividad, tienen más riesgo de desarrollar ACV.

Correspondencia:
Ignacio Madero-Cabib

Avenida Vicuña Mackenna 4860

Casilla 306, Correo 22, Macul

7820436 Santiago, Chile

E-mail: i.maderocabib@uc.cl

Recibido: 15 out 2020

Aprobado: 7 fev 2021

Cómo citar: Jerez MP,

Madero-Cabib I. Trayectorias

de estrés familiar y laboral y

su asociación con accidentes

cerebrovasculares. Rev Saude

Publica. 2021;55:101.

https://doi.org/10.11606/

s1518-8787.20210550033253

Copyright: Este es un artículo de el acceso abierto distribuido bajo la términos de la licencia Atribución Creative Commons, lo que permite el uso ilimitado, distribución y reproducción en cualquier medio, siempre que el autor y la fuente los originales se acreditan.
CONCLUSIONES: El estrés es un factor de riesgo de enfermedades cardiovasculares que experimenta de manera prolongada muchas personas en distintas etapas y dominios de la vida. En consecuencia, los sistemas de prevención de este tipo de enfermedades crónicas deben enfatizar los efectos altamente nocivos de enfrentar cotidiana y acumulativamente experiencias de vida estresantes. Esto a su vez podría mitigar las múltiples consecuencias sanitarias y financieras asociadas al ACV.

DESCRIPTORES: Accidente Cerebrovascular. Factores de Riesgo. Estrés Financiero. Agotamiento Profesional. Factores Socioeconómicos. 


\section{INTRODUCCIÓN}

Las enfermedades cardiovasculares son un grupo de desórdenes del sistema circulatorio y coronario que se manifiestan regularmente en la adultez y la vejez ${ }^{1}$, representan actualmente la principal causa de muerte en el mundo, alcanzando 17,8 millones de muertes ${ }^{2}$. Entre las enfermedades cardiovasculares, el accidente cerebrovascular (ACV) es la segunda causa de muerte en hombres y mujeres (11,9 millones de muertes $)^{2}$, y la tercera causa de discapacidad en adultos ${ }^{3}$.

Se estima que aproximadamente el $90 \%$ de los ACV pueden ser atribuidos a factores de riesgo modificables ${ }^{3}$. La evidencia epidemiológica ha destacado que entre estos factores se encuentran el hábito tabáquico, presión arterial elevada, niveles nocivos de colesterol, diabetes mellitus, obesidad, inactividad física, consumo de alcohol y patrones dietéticos inadecuados ${ }^{4}$. Sin embargo, también se ha enfatizado la creciente influencia de factores de tipo psicosocial, del entorno y del comportamiento. Entre ellos, el estrés se considera un determinante $\mathrm{crucial}^{5}$, pues se asocia al desarrollo de procesos premórbidos que conducen a la aparición de $\mathrm{ACV}^{6}$. Específicamente, las experiencias estresantes agudas y de larga duración pueden desencadenar eventos cardíacos patológicos y se asocian a un mayor riesgo de desarrollar ACV y a una mayor mortalidad asociada $\mathrm{a}^{6-9}$.

Desde la epidemiología de curso de vida, el desarrollo de ACV se ha entendido como el resultado de múltiples exposiciones a condiciones sociales, físicas y psicológicas adversas durante distintas etapas de la vida de las personas ${ }^{10-12}$. Por ejemplo, la acumulación de experiencias adversas durante la niñez, tales como enfermedades de los padres, conflictos familiares prolongados o problemas financieros, han mostrado un efecto significativamente positivo sobre el riesgo de ACV en etapas posteriores de la vida ${ }^{11}$. Por otra parte, algunas investigaciones sugieren que enfrentar eventos estresantes durante la adultez, ya sea terremotos ${ }^{13} \mathrm{o}$ eventos familiares e interpersonales como la pérdida de la pareja, así como el aislamiento comunitario, inciden en un aumento de $\mathrm{ACV}^{8,14}$.

Además de las experiencias familiares estresantes, se ha descrito que otro de los dominios en el que las personas suelen enfrentar situaciones de estrés es el mercado laboral. La evidencia sugiere que aquellas personas expuestas a múltiples estresores laborales, por ejemplo una alta carga laboral y jornadas extensas de trabajo, presentarían mayor riesgo de desarrollar ACV, especialmente cuando las exposiciones a estrés son frecuentes en el tiempo ${ }^{15}$.

A pesar de los avances en relación al efecto de estresores familiares y laborales sobre el riesgo de $\mathrm{ACV}$, existen al menos tres aspectos que aún requieren mayor elaboración en este campo de estudio. En primer lugar, hasta el momento la mayoría de las investigaciones consideran mediciones del estrés y de ACV en una o dos etapas de la vida. Esto impide entender patrones de estrés de tipo familiar o laboral a lo largo de la vida de las personas, así como su efecto acumulativo sobre el ACV.

En segundo lugar, pese a la creciente evidencia sobre las consecuencias de estresores familiares y laborales sobre ACV, la mayoría de los estudios se han centrado en uno u otro dominio por separado para medir la presencia de estrés. En consecuencia, se ha ignorado el impacto simultáneo de experimentar estrés familiar y laboral sobre este tipo de patologías. El efecto simultáneo de estrés por causas familiares y laborales es relevante por cuanto el efecto nocivo de la presencia de estrés en uno de estos dominios podría ser compensado por la ausencia de estrés en el otro; o por el contrario, el efecto sobre el ACV podría verse aumentado por la interacción de la presencia de estrés en ambos dominios de vida.

En tercer lugar, la creciente investigación en este campo ha sido conducida casi únicamente en países de altos ingresos, los que cuentan con sistemas sanitarios mejores y orientados a la promoción de la salud y prevención de enfermedades, tales como Finlandia y Reino Unido ${ }^{8,15}$. Luego de revisar exhaustivamente la evidencia actual, no se encontraron estudios sobre trayectorias de estrés laboral o familiar y ACV en países en desarrollo o de ingresos medios, como aquellos de la región latinoamericana. Comprender la relación entre trayectorias de 
estrés y ACV podría permitir reorientar las políticas públicas de prevención de este tipo de enfermedades también en los países latinoamericanos, dirigiendo esfuerzos hacia la mitigación del estrés en diferentes dominios y etapas de la vida de las personas.

Considerando estas brechas de conocimiento, el presente estudio se plantea dos objetivos principales: Reconstruir diferentes tipos de trayectorias de estrés simultáneo en el dominio familiar y laboral durante la vida de las personas en Chile; y estimar la asociación de dichos tipos de trayectorias sobre la presencia de ACV en la vejez.

\section{MÉTODOS}

\section{Base de Datos}

Este estudio utilizó datos de la encuesta "Curso de vida y vulnerabilidad en personas mayores en Santiago, Chile", la cual recolectó información retrospectiva en múltiples dominios de la vida, tales como historias residenciales, educacionales y ocupacionales, hábitos de riesgo para la salud, patrones maritales y de fertilidad, vulnerabilidades financieras, y estados de salud en la vejez. La encuesta fue cara a cara, aplicada entre marzo y agosto del año 2019, y obtuvo información representativa de 802 personas de 65 a 75 años de edad que residían en Santiago de Chile.

La recopilación de datos se realizó siguiendo los estándares de calidad definidos por la Asociación Americana de Investigación de Opinión Pública ${ }^{16}$. La selección de la muestra de individuos fue aleatoria, con una varianza máxima $(\mathrm{p}=0,5)$, un nivel de confianza del 95\%, y un error estimado de $\pm 3,5$ puntos para una población infinita. Para mitigar los posibles sesgos de no respuesta en la selección de la muestra, la base de datos fue ajustada por un factor de ponderación que corrige nuestras estimaciones por características poblacionales conocidas de este tramo de edad en Santiago, tales como zonas de residencia, nivel educacional y género.

Para recopilar los datos se utilizó el instrumento retrospectivo llamado "calendario de curso de vida"17,18, diseñado especialmente para ayudar a los entrevistados a recordar y organizar cronológicamente los episodios a lo largo de sus vidas con fechas aproximadas de ocurrencia. En la Figura 1 presentamos un ejemplo del calendario de curso de vida utilizado en esta investigación. La unidad de tiempo empleada en esta herramienta correspondió a información anual. Dado que una limitante importante de los instrumentos de medición retrospectivos corresponde al sesgo de memoria, el calendario de curso de vida utilizado en este estudio fue creado abordando tres aspectos claves del funcionamiento de la memoria autobiográfica. Primero, incluye una línea de tiempo que fuerza a las personas a pensar retrospectivamente sobre sus vidas de forma cronológica. Segundo, exige a los individuos que recuerden en primer lugar experiencias en dominios clave para la vida (por ejemplo, nacimientos, matrimonios, divorcios, viudez) para luego relacionarlos con otras experiencias en el resto de los dominios, aumentando así la precisión de la reconstrucción del pasado. Y tercero, entrega a los encuestados la oportunidad de agregar información al calendario, incluso si esto significa retroceder o avanzar en la línea de tiempo ${ }^{17,18}$.

\section{Variables}

\section{Variable Independiente: Trayectorias de estrés familiar y laboral}

Para reconstruir tipos de trayectorias de estrés familiar y laboral, se consideraron preguntas del calendario de curso de vida en las que los participantes debieron identificar períodos de sus vidas en que experimentaron estrés por motivos familiares y laborales de acuerdo a su propio criterio. Específicamente, mientras que para el dominio familiar a cada participante se le solicitó marcar los años en que "sintió estrés por causas familiares", en el dominio laboral se le pidió que para cada uno de los trabajos en los que se había desempeñado durante su vida respondiera "sí” o "no" a la pregunta “En general fue un trabajo estresante?”. A partir de estas preguntas, se creó en primer lugar la variable “estrés laboral”, que midió para cada año 


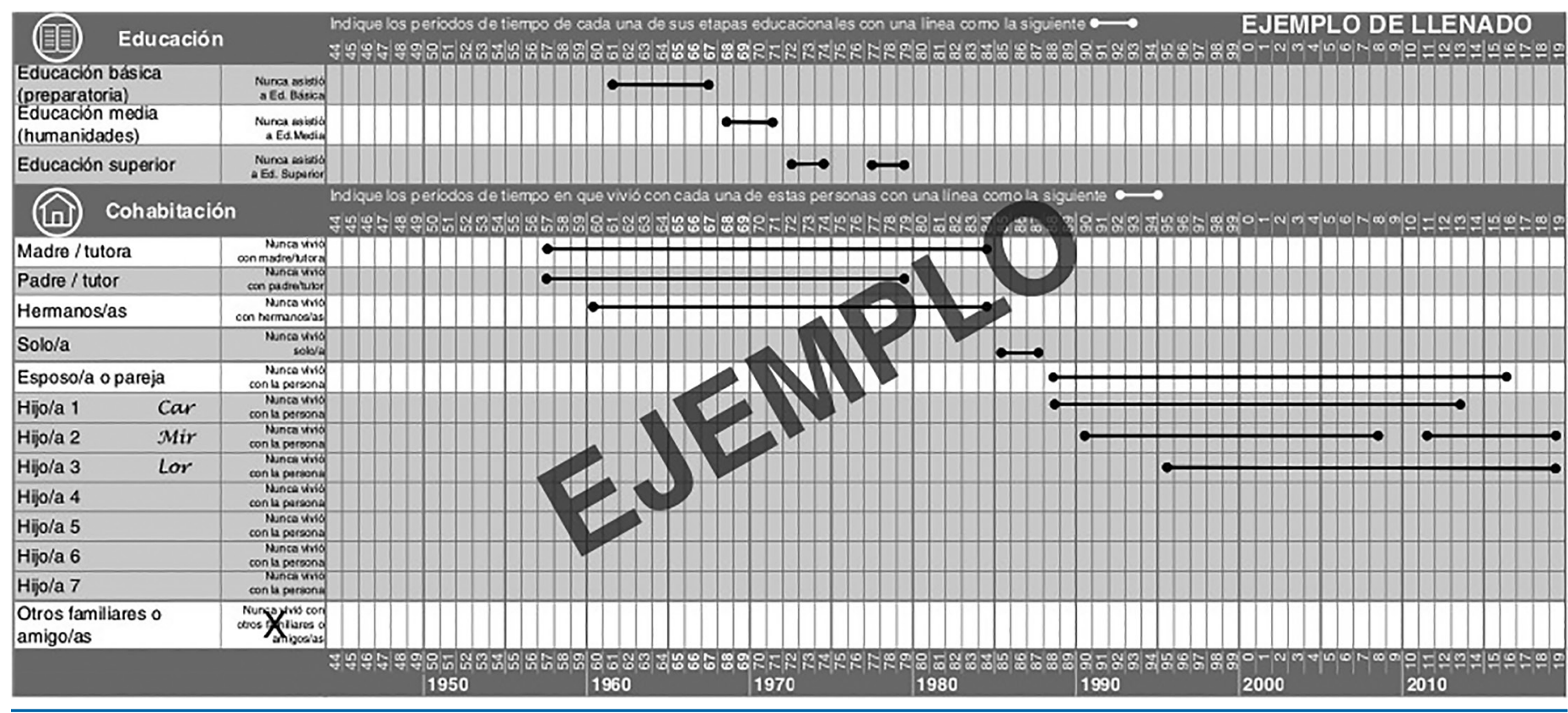

Figura 1. Calendario de curso de vida utilizado en este estudio.

de vida de las personas las categorías "presencia de estrés", "ausencia de estrés" o "fuera del mercado laboral" en caso de ser un año en el que la persona no se encontrara empleada. En segundo lugar, se creó la variable "estrés familiar", la cual de modo similar identificó para cada año de vida de las personas las categorías: "presencia de estrés" y "ausencia de estrés".

\section{Variable Dependiente: Accidente cerebrovascular}

La variable dependiente de nuestro estudio corresponde a haber experimentado o no un accidente cerebrovascular. Siguiendo estándares internacionales, a los participantes se les consultó “'Alguna vez un médico le ha dicho que tiene o tenía alguno de los problemas de salud que figuran en esta tarjeta? Recuerde que pueden ser problemas de salud que tiene actualmente, o tuvo anteriormente". Entre las opciones se encontraba "Derrame cerebral o enfermedad vascular cerebral".

\section{Variables de Control: Factores sociodemográficos y de riesgo tradicional de ACV}

Tomando en cuenta estudios realizados en países de altos ingresos, los análisis fueron ajustados primero por las siguientes cuatro variables sociodemográficas: edad, género (mujer, hombre), nivel educacional (primaria, secundaria o terciaria), y cobertura de salud (pública, privada). Esta última variable se considera un indicador proxy de nivel socioeconómico del entrevistado por cuanto las personas con mejor situación financiera acceden a cobertura privada de salud. Nuestros análisis se ajustaron también por factores de riesgo tradicionales de $\mathrm{ACV}$, tales como índice de masa corporal (obeso, no obeso); actividad física (una o más veces por semana / una vez al mes o menos); frecuencia de consumo de (1) lácteos, (2) legumbres, alubias o huevos y (3) carne, pescado o ave (una o más veces por semana / menos de una vez por semana); hábito tabáquico actual (presencia/ausencia); y diagnóstico de hipertensión (presencia/ausencia).

\section{Métodos Estadísticos}

En primer lugar, empleamos un análisis de secuencia multicanal ${ }^{19-21}$ para reconstruir tipos representativos de trayectorias simultáneas de estrés familiar y laboral a lo largo de la vida. El análisis de secuencia multicanal permite comparar similitudes y diferencias entre trayectorias de vida en dos o más dominios de la vida (en este caso estrés laboral y estrés familiar), por medio del cálculo de la distancia entre dichas trayectorias. La distancia se entiende como el costo de transformar la trayectoria de un individuo en un dominio 
específico a la trayectoria de otro individuo en dicho dominio, mediante dos operaciones concretas: substitución de estatus a partir de los que se miden las trayectorias, e inserción o eliminación de estos estatus ${ }^{22}$.

Cuando se obtienen las distancias entre las distintas trayectorias individuales, estas son introducidas en una matriz de distancias, sobre la cual se realiza un análisis de conglomerados jerárquico usando el método de Ward ${ }^{23}$, que nos permitió agrupar trayectorias similares entre sí (vale decir, con menor distancia entre ellas) en diferentes conglomerados o tipos representativos de trayectorias de estrés familiar y laboral. Finalmente, para determinar el número más robusto de tipos de trayectorias representativos de la diversidad de trayectorias posibles en la muestra, se utilizaron cuatro criterios estadísticos de selección: average silhouette width (ASW), point biserial correlation (PBS), Hubert's gamma (HG) y Hubert's C (HC) $)^{24}$. Los puntajes de estos índices varían de -1 a 1, o de 0 a 1 . Para asegurar la comparación entre los criterios, sus puntajes fueron estandarizados. En el caso de ASW, $\mathrm{PBC}$ y HG, valores mayores significan una mejor solución, mientras que valores menores en HC indican una mejor solución.

Una vez identificado el número de tipos más robusto de trayectoria de estrés familiar y laboral, este se convirtió en la principal variable independiente para medir la asociación con ACV en un modelo de regresión logística, el cual fue ajustado por las variables de control indicadas previamente. Los análisis de este estudio fueron conducidos en el software estadístico $\mathrm{R}^{25}$, específicamente con el paquete estadístico TraMine $R^{22}$ para estimar análisis de secuencia multicanal, y el paquete estadístico surve ${ }^{26}$ para trabajar con base de datos ponderada.

\section{RESULTADOS}

\section{Características de la Muestra}

Las proporciones univariadas ponderadas de las variables dependientes y de control se muestran en la Tabla 1. Entre los datos más destacados observamos que el 6,4\% de la muestra reportó haber experimentado ACV, las mujeres constituyeron el 56,7\% de los analizados, un 30,3\% reportó obesidad, y un 66\% presencia de un diagnóstico de hipertensión.

Tabla 1. Proporciones univariadas ponderadas de variables dependientes y de control.

\begin{tabular}{|c|c|c|c|}
\hline & $\%$ & & $\%$ \\
\hline Diagnóstico ACV & \multicolumn{3}{|c|}{ Consumo de legumbres, alubias o huevos } \\
\hline Ausencia & 93,6 & Menos de una vez por semana & 3,7 \\
\hline Presencia & 6,4 & Una o más veces por semana & 96,2 \\
\hline Género & \multicolumn{3}{|c|}{ Actividad física } \\
\hline Hombre & 43,3 & Una vez al mes o menos & 56,6 \\
\hline Mujer & 56,7 & Una o más veces por semana & 43,4 \\
\hline Nivel educacional & \multicolumn{3}{|c|}{ Hábito tabáquico } \\
\hline Secundaria o terciaria & 61,5 & Ausencia & 81,1 \\
\hline Primaria & 38,5 & Presencia & 17,3 \\
\hline Cobertura de salud & \multicolumn{3}{|c|}{ Hipertensión } \\
\hline Pública & 88,0 & Ausencia & 66,0 \\
\hline Privada & 11,2 & Presencia & 34,0 \\
\hline Consumo de lácteos & \multicolumn{3}{|c|}{ Índice de masa corporal } \\
\hline Menos de una vez por semana & 12,3 & No obeso & 69,7 \\
\hline Una o más veces por semana & 87,2 & Obeso & 30,3 \\
\hline \multicolumn{4}{|l|}{ Consumo de carne, pescado o ave } \\
\hline Menos de una vez por semana & 1,7 & & \\
\hline Una o más veces por semana & 98,3 & & \\
\hline
\end{tabular}

ACV: accidente cerebrovascular.

Nota: La única variable que presentó valores perdidos fue "Hábito tabáquico" con un 1,6\%. 


\section{Trayectorias de Estrés Familiar y Laboral}

La Figura 2 sugiere que, basados en los criterios de selección antes mencionados, cuatro conglomerados corresponden al número más robusto de tipos de trayectorias para representar adecuadamente la diversidad de patrones de estrés familiar y laboral en la muestra estudiada.

En las Figuras 3 y 4 se detallan los cuatro tipos de trayectorias en dos tipos de gráficos: gráficos de cronogramas y gráficos de secuencias individuales, respectivamente. En cada uno de estos gráficos, la trayectoria de estrés familiar y de estrés laboral se despliega en diferentes columnas. A la izquierda de los gráficos están representados los nombres y proporciones ponderados de cada tipo de trayectoria, y a la derecha los estatus de estrés familiar y laboral utilizados para reconstruir dichas trayectorias. En el gráfico de cronogramas, el eje X representa la edad de las personas; y el eje Y, la proporción (0 a 1) de personas en diferentes tipos de estatus de estrés en el tiempo. En el gráfico de secuencias individuales, el eje X también indica la edad, pero el eje Y muestra la trayectoria específica de cada individuo clasificado en un tipo de trayectoria (es decir, existen tantas líneas como personas clasificadas en dicho tipo).

El primer tipo, "Ausencia de estrés familiar, estrés laboral persistente", incluye al 54,8\% de la muestra y corresponde a personas que mayoritariamente describieron no haber sentido estrés por causas familiares, pero sí permanentemente por causas laborales. Este grupo manifiesta haber percibido estrés laboral mayoritariamente a partir de los 20 años de edad de forma constante, lo que disminuye después de los 50 años cuando comienza a aumentar progresivamente la salida del mercado laboral.

El segundo tipo de trayectoria, "Ausencia de estrés familiar, fuera del mercado laboral", reúne al 18,3\% de la muestra y se caracteriza por personas que la mayoría del tiempo en sus vidas se encontraron sin un empleo remunerado, y que no reportaron haber sentido estrés por causas familiares. En este grupo algunas personas reportan haber sufrido estrés laboral en etapas tempranas de la adultez (esto es, de 18 a 30 años de edad), pero luego salieron del mercado laboral.

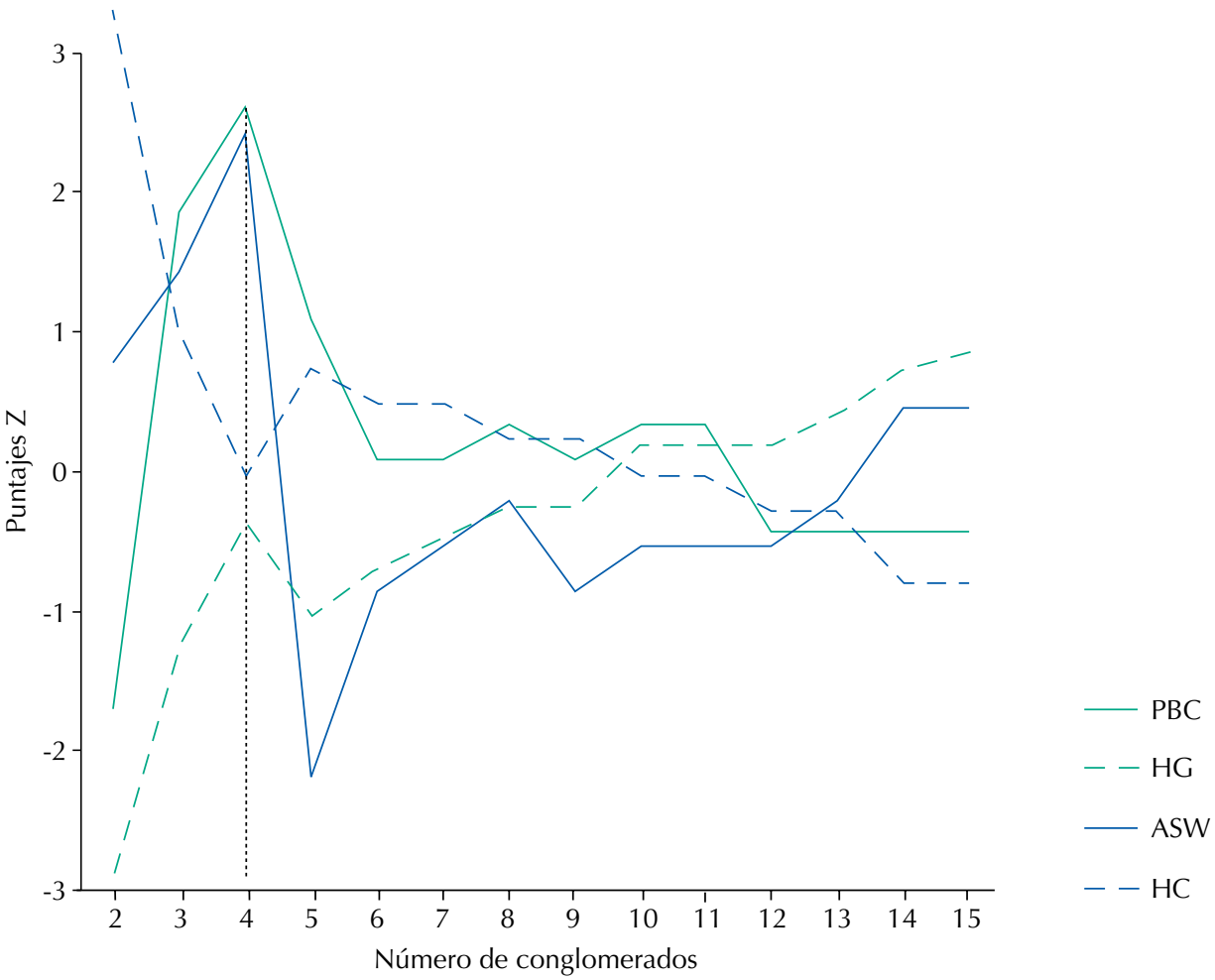

PBC: point biserial correlation; HG: Hubert's gamma; ASW: average silhouette width; HC: Hubert's C. Nota: Mayores valores para PBC, HG y ASW, y menores valores para HC, agrupan una mejor y más robusta solución para el número de conglomerados.

Figura 2. Criterios de selección de conglomerados. 


\begin{abstract}
1. Ausencia de estrés familiar, estrés laboral persistente $(54,8 \%$
\end{abstract}

2. Ausencia de estrés familiar, fuera del mercado laboral $(18,3 \%)$

3. Estrés familiar persistente, ausencia de estrés laboral $(6,9 \%)$

4. Ausencia de estrés familiar $y$ laboral (20\%)

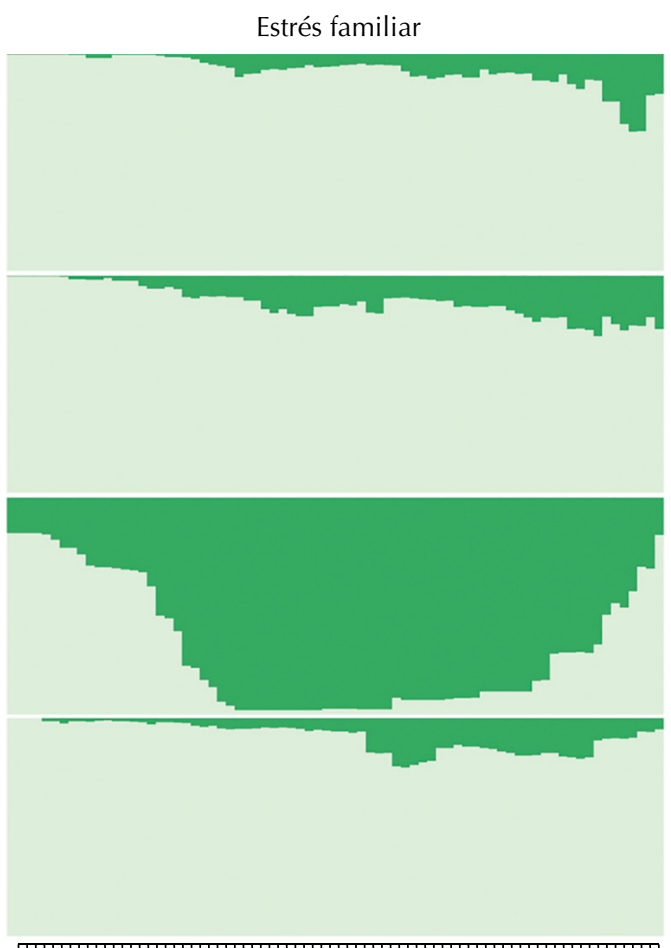

1471115192327313539434751555963677175

Estrés laboral

Estrés familiar
Estrés laboral

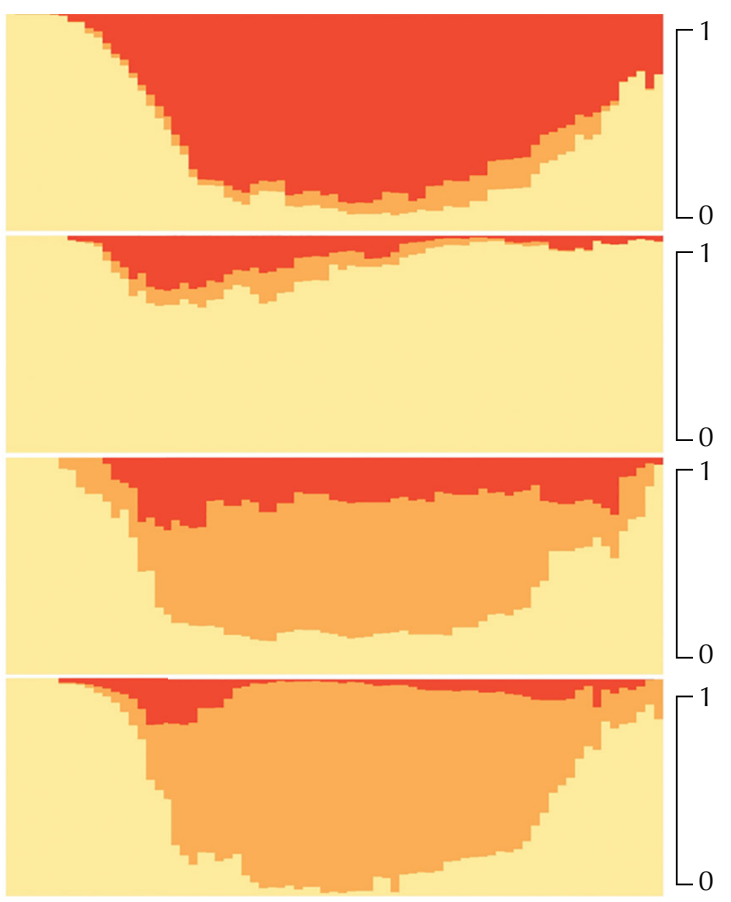

1471115192327313539434751555963677175

Fuera del mercado laboral Ausencia de estrés

Presencia de estrés

Figura 3. Gráficos de cronogramas de los cuatro tipos de trayectorias de estrés familiar y laboral.

Estrés familiar

1. Ausencia de estrés familiar, estrés laboral persistente $(54,8 \%)$

2. Ausencia de estrés familiar, fuera del mercado laboral $(18,3 \%)$

3. Estrés familiar persistente, ausencia de estrés laboral $(6,9 \%)$

4. Ausencia de estrés familiar y laboral $(20 \%)$

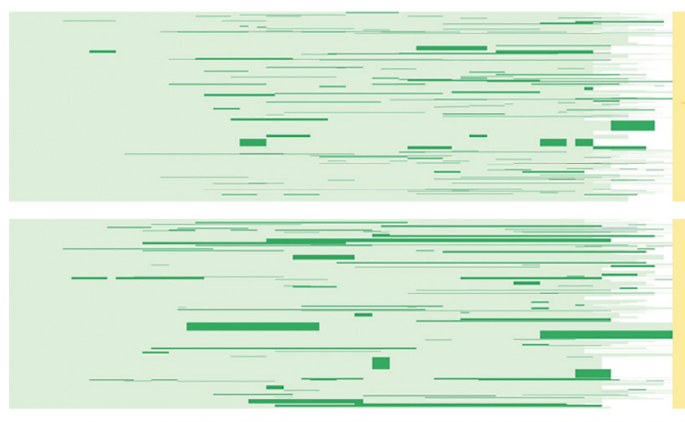

Estrés laboral
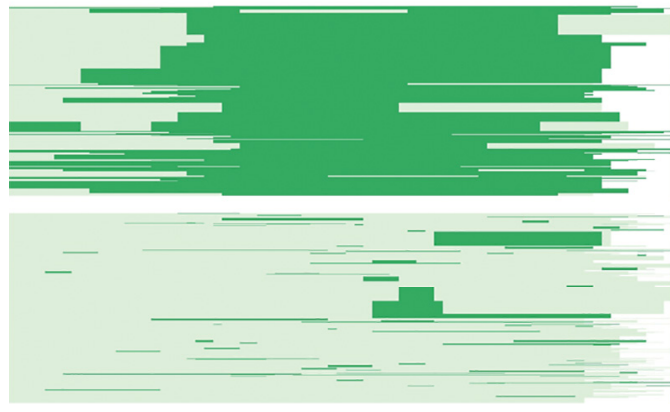

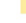
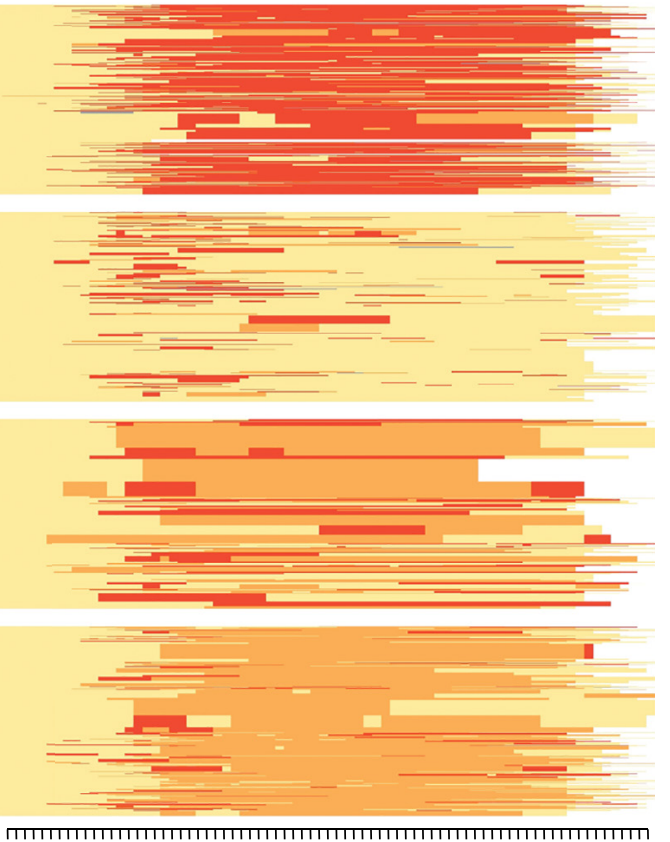

14711151923273135394347515559636771751471115192327313539434751555963677175

Estrés familiar

Estrés laboral

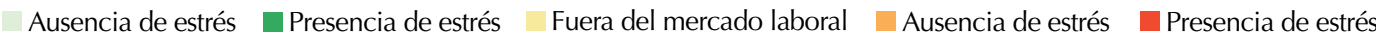

Nota: En paréntesis se indica la proporción ponderada de cada uno de los tipos de trayectoria de estrés familiar y laboral.

Figura 4. Gráficos de secuencias individuales de los cuatro tipos de trayectorias de estrés familiar y laboral. 
El tercer tipo de trayectoria, "Estrés familiar persistente, ausencia de estrés laboral", comprende el 6,9\% de la muestra y está compuesto por individuos que mayoritariamente casi no reportaron estrés por causas laborales, pero sí por causas familiares. En este tipo, se clasifican incluso algunas personas que indicaron haber sentido estrés familiar desde la primera infancia, aumentando notoriamente después de los 18 años y manteniéndose constante hasta después de los 60 años.

Tabla 2. Regresión logística sobre accidente cerebrovascular.

\begin{tabular}{|c|c|c|c|c|c|c|}
\hline & $\begin{array}{l}\text { Odds } \\
\text { ratios }\end{array}$ & $\begin{array}{l}95 \% \text { intervalos } \\
\text { de confianza }\end{array}$ & $\begin{array}{l}\text { Odds } \\
\text { ratios }\end{array}$ & $\begin{array}{l}95 \% \text { intervalos } \\
\text { de confianza }\end{array}$ & $\begin{array}{l}\text { Odds } \\
\text { ratios }\end{array}$ & $\begin{array}{c}95 \% \text { intervalos } \\
\text { de confianza }\end{array}$ \\
\hline \multicolumn{7}{|l|}{ Tipo de trayectorias de estrés laboral y familiar } \\
\hline \multicolumn{7}{|l|}{ Ausencia de estrés familiar y laboral } \\
\hline Ausencia de estrés familiar, estrés laboral persistente & 2,42 & $0,77-7,56$ & $3,16^{\mathrm{d}}$ & $0,89-11,17$ & $3,38^{\mathrm{d}}$ & $1,00-11,50$ \\
\hline Ausencia de estrés familiar, fuera de mercado laboral & $9,18^{\mathrm{b}}$ & $2,16-39,00$ & $8,54^{\mathrm{b}}$ & $1,80-40,50$ & $8,28^{\mathrm{b}}$ & $1,86-36,93$ \\
\hline Estrés familiar persistente, ausencia de estrés laboral & $12,64^{a}$ & $3,53-45,23$ & $12,90^{\mathrm{a}}$ & $2,96-56,14$ & $11,87^{\mathrm{b}}$ & $2,80-50,38$ \\
\hline \multicolumn{7}{|l|}{ Variables sociodemográficas } \\
\hline Edad & & & 1,09 & $0,87-1,36$ & 1,11 & $0,90-1,37$ \\
\hline \multicolumn{7}{|l|}{ Género } \\
\hline Hombre & - & & - & & - & \\
\hline Mujer & & & 0,94 & $0,44-2,01$ & 0,80 & $0,37-1,83$ \\
\hline \multicolumn{7}{|l|}{ Nivel educacional } \\
\hline Secundaria o terciaria & - & & - & & - & \\
\hline Primaria & & & $1,95^{\mathrm{d}}$ & $0,93-4,11$ & 1,50 & $0,79-2,84$ \\
\hline \multicolumn{7}{|l|}{ Cobertura de salud } \\
\hline Pública & - & & - & & - & \\
\hline Privada & & & $0,00^{\mathrm{a}}$ & $0,00-0,00$ & $0,00^{\mathrm{a}}$ & $0,00-0,00$ \\
\hline \multicolumn{7}{|l|}{ Factores de riesgo tradicionales de ACV } \\
\hline \multicolumn{7}{|l|}{ Índice de masa corporal } \\
\hline No obeso & - & & - & & - & \\
\hline Obeso & & & & & 1,72 & $0,74-4,00$ \\
\hline \multicolumn{7}{|l|}{ Actividad física } \\
\hline Una vez al mes o menos & - & & - & & - & \\
\hline Una o más veces por semana & & & & & 0,90 & $0,25-3,19$ \\
\hline \multicolumn{7}{|l|}{ Consumo de lácteos } \\
\hline Menos de una vez por semana & - & & - & & - & \\
\hline Una o más veces por semana & & & & & 0,51 & $0,17-1,54$ \\
\hline \multicolumn{7}{|l|}{ Consumo de legumbres, alubias o huevos } \\
\hline Menos de una vez por semana & - & & - & & - & \\
\hline Una o más veces por semana & & & & & $0,28^{c}$ & $0,09-0,86$ \\
\hline \multicolumn{7}{|l|}{ Consumo de carne, pescado o ave } \\
\hline Menos de una vez por semana & - & & - & & - & \\
\hline Una o más veces por semana & & & & & 0,76 & $0,09-6,40$ \\
\hline \multicolumn{7}{|l|}{ Hábito tabáquico } \\
\hline Ausencia & - & & - & & - & \\
\hline Presencia & & & & & 1,21 & $0,55-2,67$ \\
\hline \multicolumn{7}{|l|}{ Hipertensión } \\
\hline Ausencia & - & & - & & - & \\
\hline Presencia & & & & & 1,74 & $0,68-4,46$ \\
\hline Intercepto & 0,02 & & 0,00 & & 0,00 & \\
\hline
\end{tabular}

a $\mathrm{p}<0.001$

${ }^{\mathrm{b}} \mathrm{p}<0.01$.

${ }^{c} \mathrm{p}<0.05$.

${ }^{\mathrm{d}} \mathrm{p}<0.1$.

Nota: Efectos estadísticamente significativos marcados en gris. 
Por último, el cuarto tipo de trayectoria "Ausencia de estrés familiar y laboral" incluye al $20 \%$ de la muestra y se compone de personas que mayoritariamente describieron no haber sentido estrés ni por causas familiares ni laborales en sus vidas.

\section{Trayectorias de Estrés Familiar y Laboral y ACV}

La Tabla 2 muestra el resultado de tres modelos de regresión logística: el primero incluye solo los tipos de trayectorias de estrés, el segundo agrega las variables sociodemográficas,

Tabla 3. Regresión logística sobre accidente cerebrovascular (efectos de interacción entre tipos de trayectorias y género).

\begin{tabular}{|c|c|c|c|c|c|c|}
\hline & $\begin{array}{l}\text { Odds } \\
\text { ratios }\end{array}$ & $\begin{array}{c}95 \% \text { intervalos } \\
\text { de confianza }\end{array}$ & $\begin{array}{l}\text { Odds } \\
\text { ratios }\end{array}$ & $\begin{array}{c}95 \% \text { intervalos } \\
\text { de confianza }\end{array}$ & $\begin{array}{l}\text { Odds } \\
\text { ratios }\end{array}$ & $\begin{array}{c}95 \% \text { intervalos } \\
\text { de confianza }\end{array}$ \\
\hline \multicolumn{7}{|l|}{ Tipo de trayectorias de estrés laboral y familiar } \\
\hline Ausencia de estrés familiar y laboral & - & & - & & - & \\
\hline Ausencia de estrés familiar, estrés laboral persistente & 1,31 & $0,23-7,35$ & 1,94 & $0,28-13,18$ & 2,27 & $0,33-15,58$ \\
\hline Ausencia de estrés familiar, fuera de mercado laboral & 7,50 & $0,59-95,57$ & 7,71 & $0,47-125,29$ & 8,98 & $0,59-95,57$ \\
\hline Estrés familiar persistente, ausencia de estrés laboral & $8,32^{\mathrm{b}}$ & $1,52-45,64$ & $9,15^{\mathrm{b}}$ & $1,37-61,18$ & $8,69^{\mathrm{b}}$ & $1,22-61,83$ \\
\hline \multicolumn{7}{|l|}{ Tipo de trayectorias de estrés laboral y familiar * Género } \\
\hline Ausencia de estrés familiar, estrés laboral persistente * Mujer & 3,54 & $0,30-42,16$ & 2,72 & $0,20-36,25$ & 2,28 & $0,17-28,86$ \\
\hline Ausencia de estrés familiar, fuera de mercado laboral * Mujer & 2,27 & $0,09-46,06$ & 1,64 & $0,06-44,34$ & 1,27 & $0,04-36,28$ \\
\hline Estrés familiar persistente, ausencia de estrés laboral * Mujer & 2,06 & $0,15-33,96$ & 2,00 & $0,14-28,25$ & 1,92 & $0,12-29,51$ \\
\hline \multicolumn{7}{|l|}{ Variables sociodemográficas } \\
\hline Edad & & & 1,09 & $0,88-1,36$ & 1,12 & $0,92-1,37$ \\
\hline \multicolumn{7}{|l|}{ Nivel educacional } \\
\hline Secundaria o terciaria & - & & - & & - & \\
\hline Primaria & & & 1,87 & $0,85-4,01$ & 1,44 & $0,74-2,80$ \\
\hline \multicolumn{7}{|l|}{ Cobertura de salud } \\
\hline Pública & - & & - & & - & \\
\hline Privada & & & $0,00^{\mathrm{a}}$ & $0,00-0,00$ & $0,00^{\mathrm{a}}$ & $0,00-0,00$ \\
\hline \multicolumn{7}{|l|}{ Factores de riesgo tradicionales de ACV } \\
\hline \multicolumn{7}{|l|}{ Índice de masa corporal } \\
\hline No obeso & - & & - & & - & \\
\hline Obeso & & & & & 1,70 & $0,75-3,88$ \\
\hline \multicolumn{7}{|l|}{ Actividad física } \\
\hline Una vez al mes o menos & - & & - & & - & \\
\hline Una o más veces por semana & & & & & 0,91 & $0,26-3,25$ \\
\hline \multicolumn{7}{|l|}{ Consumo de lácteos } \\
\hline Menos de una vez por semana & - & & - & & - & \\
\hline Una o más veces por semana & & & & & 0,51 & $0,17-1,51$ \\
\hline \multicolumn{7}{|l|}{ Consumo de legumbres, alubias o huevos } \\
\hline Menos de una vez por semana & - & & - & & - & \\
\hline Una o más veces por semana & & & & & $0,29^{b}$ & $0,09-0,88$ \\
\hline \multicolumn{7}{|l|}{ Consumo de carne, pescado o ave } \\
\hline Menos de una vez por semana & - & & - & & - & \\
\hline Una o más veces por semana & & & & & 0,76 & $0,09-6,52$ \\
\hline \multicolumn{7}{|l|}{ Hábito tabáquico } \\
\hline Ausencia & - & & - & & - & \\
\hline Presencia & & & & & 1,25 & $0,58-2,69$ \\
\hline \multicolumn{7}{|l|}{ Hipertensión } \\
\hline Ausencia & - & & - & & - & \\
\hline Presencia & & & & & 1,73 & $0,68-4,40$ \\
\hline Intercepto & 0,02 & & 0,00 & & 0,00 & \\
\hline
\end{tabular}

a $p<0.001$.

${ }^{\mathrm{b}} \mathrm{p}<0.05$.

Nota: Efectos estadísticamente significativos marcados en gris. 
mientras que el tercero considera también los factores de riesgo tradicionales de ACV. Los resultados son reportados en odds ratios (OR) con sus respectivos intervalos de confianza y niveles de significancia estadística.

Como se observa en el modelo que considera los ajustes de todas las variables de control, la posibilidad de sufrir ACV es significativamente mayor en todos los tipos de trayectorias de estrés, en comparación a aquellos sujetos agrupados en la trayectoria "Ausencia de estrés familiar y laboral". Específicamente, en aquellos sujetos que componen la trayectoria "Ausencia de estrés familiar, estrés laboral persistente", la probabilidad de sufrir ACV es más de dos veces mayor $(\mathrm{OR}=3,38 ; \mathrm{p}<0,1)$. Mientras que para aquellos clasificados en la trayectoria "Ausencia de estrés familiar, fuera del mercado laboral" la probabilidad es más de siete veces mayor ( $\mathrm{OR}=8,28 ; \mathrm{p}<0,01)$, y para aquellos que siguieron la trayectoria "Estrés familiar persistente, ausencia de estrés laboral" la probabilidad es más de diez veces mayor $(\mathrm{OR}=11,87 ; \mathrm{p}<0,01)$. En relación a los factores de control, se observa que el hecho de tener cobertura privada de salud, así como el consumo de legumbres, alubias o huevos más de una vez a la semana, reduce el riesgo de ACV.

Adicionalmente, basados en evidencia previa sobre diferencias entre hombres y mujeres en enfermedades cerebrovasculares ${ }^{27}$, condujimos un análisis de sensibilidad incluyendo efectos de interacción entre los tipos de trayectorias y género sobre el riesgo de ACV (ver Tabla 3). Como se puede observar, el modelo que considera todos los controles sugiere que solo en la relación entre la trayectoria "Estrés familiar persistente, ausencia de estrés laboral” y ACV los hombres muestran un efecto significativamente superior al de las mujeres $(\mathrm{OR}=8,69 ; \mathrm{p}<0,5)$.

\section{DISCUSIÓN}

Utilizando una encuesta retrospectiva, representativa de personas de 65 a 75 años de edad de la ciudad de Santiago, Chile, y basados en un enfoque de curso de vida, este estudio analizó la asociación entre distintos tipos de trayectorias simultáneas de estrés familiar y laboral y la presencia de ACV en la vejez. Los resultados de este estudio indican que una proporción importante de personas mayores en Chile $(61,7 \%)$ siguieron trayectorias marcadas por la presencia permanente de estrés familiar y/o laboral. Asimismo, una proporción no menor $(18,3 \%)$ tuvo una trayectoria caracterizada por la ausencia prolongada del mercado laboral.

En términos de la asociación con ACV, observamos que trayectorias caracterizadas tanto por la exposición permanente a situaciones familiares y/o laborales estresantes a lo largo de la vida, se asocian significativamente con la presencia de esta enfermedad cardiovascular. Estos hallazgos son coherentes con estudios transversales o cros-seccionales previos. Por ejemplo, en un metaanálisis de 14 estudios que involucró 10.130 pacientes con diagnóstico de ACV, se estimó un aumento del riesgo del 33\% en esta patología entre aquellos que reportaron sentir estrés por motivos familiares, laborales o financieros ${ }^{28}$.

Nuestros resultados muestran en particular que el tipo de trayectoria caracterizado por la presencia persistente de estrés vinculado a causas familiares, pero con ausencia de estrés laboral, se asocia más a la presencia de ACV (indicando un riesgo más de 10 veces superior al tipo de trayectoria "Ausencia de estrés familiar y laboral"). Este resultado se ajusta adecuadamente a estudios previos que han observado un efecto relevante sobre la presencia de $\mathrm{ACV}$ en aquellas personas que enfrentan relaciones maritales e interpersonales estresantes ${ }^{29}$. Además, nuestros análisis de sensibilidad (Tabla 3) nos muestran que este tipo de trayectoria es significativamente más riesgosa en hombres que en mujeres. Al respecto, algunas investigaciones han apuntado un aumento del riesgo de sufrir ACV en grupos que habían experimentado estrés asociado al divorcio o fallecimiento del cónyuge, particularmente entre hombres ${ }^{14}$.

Por otro lado, nuestro estudio evidencia una fuerte asociación con ACV entre personas que reportan no haber sufrido estrés por causas familiares, pero estaban fuera del 
mercado laboral durante períodos prolongados. Estos resultados también coinciden con estudios transversales previos, que muestran que aquellas personas que dejan de trabajar remuneradamente tienen más del doble de riesgo de padecer ACV (así como otras enfermedades cardiovasculares como el infarto agudo al miocardio), especialmente si la pérdida del empleo se produce de forma involuntaria ${ }^{27,30,31}$. Además, otros estudios en Latinoamérica indican una mayor mortalidad asociada a ACV en sujetos inactivos ${ }^{32}$. Una posible explicación de esta asociación corresponde a que, sobre todo en países como Chile, tener un empleo de manera continua implica obtener a un ingreso financiero que permite satisfacer necesidades individuales tales como el acceso a mejores servicios preventivos de salud y a tratamientos farmacológicos ${ }^{33-35}$.

\section{Limitaciones}

Este estudio tiene ciertas limitaciones que deben ser consideradas. En primer lugar, debido al ineludible sesgo de memoria, los encuestados podrían haber reportado de forma inexacta experiencias tempranas de su vida. Para mitigar este sesgo, el calendario de curso de vida empleado en este estudio incorporó innovaciones metodológicas orientadas a fortalecer la memoria autobiográfica, tales como la descomposición de los dominios de vida en diferentes subcategorías, el uso de ayudas visuales y la orientación de un encuestador debidamente capacitado en el proceso de rellenado del cuestionario. En relación a la muestra, es relevante considerar que esta es representativa de la capital, pero no de todo Chile. Esto es importante, por cuanto el centralismo del país genera que el acceso a los servicios de salud sea mayor y de mejor calidad en la capital que en el resto de las regiones. Una última limitación relevante refiere a que el reporte de estrés por parte de los encuestados depende estrechamente de su percepción del concepto "estrés". Sabemos, sin embargo, que no existe una definición única de este término, pues abarca aspectos psicológicos, emocionales, motivacionales y cognitivos. Asimismo, al momento de identificar estrés laboral, los participantes pueden haber considerado aspectos diferentes que cuando reportaron estrés familiar.

\section{IMPLICANCIAS Y CONCLUSIÓN}

Este estudio tiene implicancias relevantes para el campo de enfermedades cardiovasculares. Los ACV son patologías de alta prevalencia, con consecuencias catastróficas en la salud y altamente asociadas a factores de riesgo modificables, entre ellos el estrés. Para disminuir la mortalidad y las secuelas que estas patologías tienen en la población, es fundamental considerar dichos factores de riesgo y fortalecer los sistemas sanitarios con un enfoque promocional, preventivo, con eje en la disminución de las inequidades en salud, y no solo en la optimización de tecnologías médicas de tratamiento y rehabilitación.

Como sugiere este estudio, el estrés es un factor de riesgo que experimenta de manera prolongada una alta proporción de personas en distintas etapas y dominios de la vida. En consecuencia, es necesario adoptar un enfoque promocional y preventivo que enfatice los efectos adversos de experimentar cotidiana y acumulativamente situaciones estresantes. La formulación de políticas públicas orientadas a reducir el estrés podría contribuir a mitigar el riesgo de ACV en poblaciones tradicionalmente susceptibles.

\section{REFERENCIAS}

1. Melo-Silva AM, Mambrini JVM, Souza Junior PRB, Andrade FB, Lima-Costa MF. Hospitalizations among older adults: results from ELSI-Brazil. Rev Saude Publica. 2018;52 Suppl 2:3s. https://doi.org/10.11606/S1518-8787.2018052000639

2. GBD 2017 Causes of Death Collaborators. Global, regional, and national age-sex-specific mortality for 282 causes of death in 195 countries and territories, 1980-2017: a systematic analysis for the Global Burden of Disease Study 2017. Lancet. 2018;392(10159):1736-88. https://doi.org/10.1016/S0140-6736(18)32203-7 
3. Campbell BCV, Khatri P. Stroke. Lancet. 2020;396(10244):129-42. https://doi.org/10.1016/S0140-6736(20)31179-X

4. Joseph P, Leong D, McKee M, Anand SS, Schwalm JD, Teo K, et al. Reducing the Global Burden of Cardiovascular Disease, Part 1: the epidemiology and risk factors. Circ Res. 2017;121(6):677-94. https://doi.org/10.1161/CIRCRESAHA.117.308903

5. Surkan PJ, Sakyi KS, Hu A, Olinto MT, Gonçalves H, Horta BL, et al. Impact of stressful life events on central adiposity in the Pelotas Birth Cohort. Rev Saude Publica. 2018;52:61. https://doi.org/10.11606/s1518-8787.2018052000161

6. Máximo RO, Lopes IC, Brigola AG, Luchesi BM, Gratão ACM, Inouye K, et al. Pre-frailty, frailty and associated factors in older caregivers of older adults. Rev Saude Publica. 2020;54:17. https://doi.org/10.11606/s1518-8787.2020054001655

7. Lightbody CE, Clegg A, Patel K, Lucas JC, Storey H, Hackett ML, et al. Systematic review and meta-analysis of psychosocial risk factors for stroke. Semin Neurol. 2017;37(3):294-306. https://doi.org/10.1055/s-0037-1603758

8. Kivimäki M, Steptoe A. Effects of stress on the development and progression of cardiovascular disease. Nat Rev Cardiol. 2018;15(4):215-29. https://doi.org/10.1038/nrcardio.2017.189

9. Kotlęga D, Gołąb-Janowska M, Masztalewicz M, Ciećwież S, Nowacki P. The emotional stress and risk of ischemic stroke. Neurol Neurochir Pol. 2016;50(4):265-70. https://doi.org/10.1016/j.pjnns.2016.03.006

10. Blanco M. El enfoque del curso de vida: orígenes y desarrollo. Rev Lat Am Población.2011;5(1):5-31. https://doi.org/10.31406/relap2011.v5.i1.n8.1

11. Kuh D, Schlomo YB, Ezzra S. A life course approach to chronic disease epidemiology. Oxford (UK): Oxford University Press; 2004.

12. Kornerup H, Osler M, Boysen G, Barefoot J, Schnohr P, et al. Major life events increase the risk of stroke but not of myocardial infarction: results from the Copenhagen City Heart Study. Eur J Cardiovasc Prev Rehabil. 2010;17(1):113-8. https://doi.org/10.1097/HJR.0b013e3283359c18

13. Ripoll Gallardo A, Pacelli B, Alesina M, Serrone D, lacutone G, Faggiano F, et al. Medium- and long-term health effects of earthquakes in high-income countries: a systematic review and meta-analysis. Int J Epidemiol. 2018;47(4):1317-32. https://doi.org/10.1093/ije/dyy130

14. Engström G, Khan FA, Zia E, Jerntorp I, Pessah-Rasmussen H, Norrving B, et al. Marital dissolution is followed by an increased incidence of stroke. Cerebrovasc Dis (Base). 2004;18(4):318-24. https://doi.org/10.1159/000080770

15. Li J, Loerbroks A, Bosma H, Angerer P. Work stress and cardiovascular disease: a life course perspective. J Occup Health. 2016;58(2):216-9. https://doi.org/10.1539/joh.15-0326-OP

16. American Association for Public Opinion Research. Standard definitions. Final dispositions of case codes and outcome rates for surveys. Washington, DC: AAPOR; 2016 [citado 15 Abr 2016]. Disponible en: https://www.aapor.org/Standards-Ethics/Standard-Definitions-(1).aspx

17. Morselli D, Dasoki N, Gabriel R, Gauthier JA, Henke J, Le Goff JM. Using life history calendars to survey vulnerability. In: Oris M, Roberts C, Joye D, Ernst Stähli M, editors. Surveying human vulnerabilities across the life course. Cham $(\mathrm{CH})$ : Springer; 2016. p.177-201. (Life Course Research and Social Policies; $\mathrm{n}^{\circ}$ 3).

18. Morselli D, Le Goff JM, Gauthier JA. Self-administered event history calendars: a possibility for surveys? Contemp Soc Sci. 2019;14(3-4):423-46. https://doi.org/10.1080/21582041.2017.1418528

19. Gauthier JA, Widmer ED, Bucher P, Notredame C. Multichannel sequence analysis applied to social science data. Sociol Methodol. 2010;40(1):1-38. https://www.jstor.org/stable/41336881

20. Madero-Cabib I, Fasang AE. Gendered work-family life courses and financial well-being in retirement. Adv Life Course Res. 2016;27:43-60. https://doi.org/10.1016/j.alcr.2015.11.003

21. Madero-Cabib I, Gauthier JA, Le Goff JM. The influence of interlocked employmentfamily trajectories on retirement timing. Work Aging Retire. 2016:2(1):38-53. https://doi.org/10.1093/workar/wav023

22. Gabadinho A, Ritschard G, Mueller NS, Studer M. Analyzing and visualizing state sequences in R with TraMineR. J Stat Softw. 2011;40(4):1-37. https://doi.org/10.18637/jss.v040.i04

23. Ward JH Jr. Hierarchical grouping to optimize an objective function. J Am Stat Assoc. 1963;58(301):236-44. https://doi.org/10.1080/01621459.1963.10500845 
24. Studer M. WeightedCluster library manual: a practical guide to creating typologies of trajectories in the social sciences with R. Lives Working Papers. 2013;2013(24):1-32. https://doi.org/10.12682/lives.2296-1658.2013.24

25. R Development Core Team. R: a language and environment for statistical computing. Vienna, AT: R Foundation for Statistical Computing; 2019.

26. Lumley T. Complex surveys: a guide to analysis using R. Hoboken, NJ: John Wiley \& Sons; 2011.

27. Kang MY, Kim, HR. Association between voluntary/involuntary job loss and the development of stroke or cardiovascular disease: a prospective study of middle-aged to older workers in a rapidly developing Asian country. PloS One. 2014;9(11):e113495. https://doi.org/10.1371/journal.pone.0113495

28. Booth J, Connelly L, Lawrence M, Chalmers C, Joice S, Becker C, et al. Evidence of perceived psychosocial stress as a risk factor for stroke in adults: a meta-analysis. BMC Neurol. 2015;15:233. https://doi.org/10.1186/s12883-015-0456-4

29. Egido JA, Castillo O, Roig B, Sanz I, Herrero MR, Garay MT, et al. Is psycho-physical stress a risk factor for stroke? A case-control study.J Neurol Neurosurg Psychiatry. 2012;83(11):1104-10. https://doi.org/10.1136/jnnp-2012-302420

30. Gallo WT, Teng HM, Falba TA., Kasl SV, Krumholz HM, Bradley EH. The impact of late career job loss on myocardial infarction and stroke: a 10 year follow up using the health and retirement survey. Occup Environ Med. 2006;63(10):683-7. https://doi.org/10.1136/oem.2006.02682

31. Dupre ME, George LK, Liu G, Peterson ED. The cumulative effect of unemployment on risks for acute myocardial infarction. Arch Intern Med. 2012;172(22):1731-7. https://doi.org/10.1001/2013.jamainternmed.447

32. Sposato LA, Ioli P, Povedano G, Esnaola y Rojas MM, Saposnik G; Argentinean Neurological Society; ReNACer Investigators. Unemployment: a social risk factor associated with early ischemic stroke mortality? Results from the Argentinean National Stroke Registry (ReNACer). J Stroke Cerebrovasc Dis. 2012;21(8):679-83. https://doi.org/10.1016/j.jstrokecerebrovasdis.2011.02.018

33. Madero-Cabib I, Biehl A, Sehnbruch K, Calvo E, Bertranou F. Private pension systems built on precarious foundations: a cohort study of labor-force trajectories in Chile. Res Aging. 2019;41(10):961-87. https://doi.org/10.1177/0164027519874687

34. Madero-Cabib I, Undurraga R, Valenzuela C. How have women's employment patterns during young adulthood changed in Chile? A cohort study. Longit Life Course Stud. 2019;10(3):375-97. https://doi.org/10.1332/175795919X15628474680736

35. Madero-Cabib I, Corna L, Baumann I. Aging in different welfare contexts: a comparative perspective on later-life employment and health. J Gerontol B Psychol Sci Soc Sci. 2020;75(7):1515-26. https://doi.org/10.1093/geronb/gbz037

Financiamiento: Esta investigación ha recibido el apoyo financiero de tres fondos de investigación de la Agencia Nacional de Investigación y Desarrollo (ANID) en Chile: ANID/FONDECYT/INICIACION/No 11180360; ANID/FONDAP/No 15130009; ANID/Millennium Science Initiative/Grant NCS17_062 "Millennium Nucleus for the Study of the Life Course and Vulnerability (MLIV)".

Participación de los Autores: Diseño y planificación del estudio: MPJ, IMC. Recopilación, análisis e interpretación de los datos: IMC. Redacción o revisión del manuscrito: MPJ, IMC. Aprobación de la versión final: IMC. Responsabilidad pública por el contenido del artículo: MPJ, IMC.

Conflicto de Intereses: Los autores declaran no haber conflicto de intereses. 